\title{
Novel therapeutic modalities and drug delivery in pancreatic cancer - an ongoing search for improved efficacy
}

\author{
Yuqing Zhang ${ }^{1}$, Kei Satoh ${ }^{2}$, and Min Li ${ }^{2}$ \\ 'Department of Cancer Biology, University of Texas MD Anderson Cancer Center, Houston, TX, USA; ${ }^{2}$ The Vivian L. Smith Department of Neurosurgery, Department \\ of Integrative Biology \& Pharmacology, the University of Texas Medical School at Houston, Houston, TX, USA
}

\begin{abstract}
Pancreatic cancer is an incredibly challenging disease due to its high rates of resistance to traditional chemotherapy and radiotherapy. There has been little improvement in the prognosis of pancreatic cancer cases in the past decades, highlighting the crucial need for more effective therapeutic approaches. Erlotinib, an EGFR inhibitor, and gemcitabine, a nucleoside analog, are currently used in combination for chemotherapy treatment, but new developments in drug delivery systems using liposomes and nanoparticles may be promising new modalities for management of the disease. In addition to standard chemotherapeutic drugs, these delivery systems can be utilized to deliver therapeutic agents such as siRNA, oncolytic viruses, small molecule inhibitors, antibodies, and suicide genes. Further work is required to elucidate how ligands and antibodies could be used to enhance the targeted delivery of drugs, thus increasing specificity, improving stability, and reducing the effect of the drugs on healthy tissue. Despite significant preclinical data, there are currently very few clinical trials involving pancreatic cancer targeted drug delivery. This article summarizes current developments in targeted pancreatic cancer drug delivery, focusing on delivery systems, targets, and therapeutic agents.
\end{abstract}

Keywords: pancreatic cancer, EGFR, monoclonal antibodies, erlotinib, gemcitabine, FOLFIRINOX, siRNA, shRNA, p53, Rb, p21, p16, KRAS, liposomes, nanoparticles, suicide genes, oncolytic viruses, VEGFR, MUC1, mesothelin, carbon nanotubes

Citation

Provenance

Dates

Copyright

Correspondence address

Email

Competing interests
Zhang Y, Satoh K and Li M. Novel therapeutic modalities and drug delivery in pancreatic cancer - an ongoing search for improved efficacy Drugs in Context 2012: 212244. doi: 10.7573/dic.212244

Invited; externally peer reviewed

Submitted: 20 September 2012; Accepted, subject to peer review: 27 September 2012; Published: 11 December 2012

(C) 2012 Zhang Y, Satoh K and Li M. This is an open-access article distributed under the terms of the Creative Commons Attribution License (CC-BY-NC-ND 3.0) which allows unrestricted sharing, copying and distribution for personal use provided it is properly attributed. Commercial use is not permitted.

Min Li, PhD, The Vivian L. Smith Department of Neurosurgery, Department of Integrative Biology \& Pharmacology, The University of Texas Medical School at Houston, 6431 Fannin Street, MSE R266, Houston, TX 77030, USA

Min.Li@uth.tmc.edu

The Authors have declared that there are no competing interests

\section{Introduction}

Despite current advances in cancer screening, diagnosis, and therapeutics, pancreatic cancer remains a highly malignant disease with a dismal 5-year survival rate of $<5 \%$ [1]. In 2012, approximately 44,000 people in the United States will be diagnosed with pancreatic cancer with more than 37,000 expected to die from this disease, making pancreatic cancer the fourth leading cause of cancer-related deaths in the country [2]. Pancreatic cancer is often diagnosed at an advanced stage owing to nonspecific symptoms and a lack of reliable biomarkers. Because of the high number of locally advanced and metastatic cases at the time of diagnosis, only $15-20 \%$ of patients can undergo potentially curative surgical resection [3]. In addition, response to chemotherapy and radiotherapy is often very poor, making management of the disease extremely challenging for clinicians.

The current standard therapy for metastatic pancreatic cancer is gemcitabine-based chemotherapy. Resistance to the drug is widespread and may be due to a number of factors including poor drug delivery and inherent chemoresistance of pancreatic tumor cells. FOLFIRINOX - combination chemotherapy with leucovorin, fluorouracil, irinotecan, and oxaliplatin - is an emerging treatment that offers a better survival outcome compared to gemcitabine, albeit with increased toxicity. In a study of 342 patients, FOLFIRINOX treatment resulted in a median 11.1-month overall survival compared to 6.8 months for gemcitabine treatment [4]. It is clear that novel chemotherapeutic approaches are necessary to improve the clinical outcomes of patients with this devastating disease. For these strategies to be viable for clinical use, the development of efficacious, targeted delivery modalities will be crucial [5]. This article summarizes recent literature (1995 to 2012) and clinical trials highlighting the current progress on new therapeutic agents and drug delivery methods, and the potential targets for the treatment of pancreatic cancer.

\section{Potential alternatives to gemcitabine}

A number of therapeutic agents and drugs may serve as alternative molecular approaches to conventional chemotherapies (Table 1). Among these agents are small-molecule inhibitors, oncolytic viruses, monoclonal antibodies, suicide genes, and 
Table 1. Potential pancreatic cancer therapeutic agents.

\begin{tabular}{ll}
\hline Therapeutic agents & Targets and representative drugs \\
\hline Small-molecule inhibitors & $\begin{array}{l}\text { Erlotinib, sonic hedgehog }(\mathrm{SHH}) \text { inhibitors, } \\
\text { etc }[6,7]\end{array}$ \\
Oncolytic viruses & $\begin{array}{l}\text { Adenovirus and herpes simplex virus [5] } \\
\text { Gene therapy }\end{array}$ \\
Antibodies & EGFR, REG $p 21, p 16, K R A S$, and $b c 1-2[8]$ \\
RNA interference & ZIP4, PDX-1, etc $[9,10]$
\end{tabular}

Abbreviations

MUC1, mucin 1

doi: 10.7573/dic.212244.t001

small interfering RNA (siRNA) that target abnormal gene expression, cell-surface receptors, and cell-signaling pathways. Studies of these alternative agents may generate improved therapeutic strategies and facilitate progress towards improving the clinical outcome of pancreatic cancer patients.

Through gene therapy, cancer cell growth can be inhibited by replacing or silencing key genes in pancreatic cancer cell growth pathways involving $p 53$, retinoblastoma protein $(R b)$, p21, p16, KRAS, and bcl-2. Modulation of KRAS expression has been shown to inhibit growth of pancreatic cancer cells in vitro and growth of intraperitoneal tumors in xenograft nude mice in vivo [8]. Research has also demonstrated that re-expression of the tumor suppressor gene $p 53$ in a subcutaneous nude mouse model inhibits pancreatic tumor growth [11]. siRNA and short hairpin RNA (shRNA) can be utilized to post-transcriptionally silence gene expression in a sequencespecific manner. These molecules are being investigated in cancer biology and other fields as powerful tools for regulating expression of genes essential for cellular processes such as survival, proliferation, and drug resistance. For example, sphingosine kinase-1-targeted siRNA increased gemcitabine sensitivity in pancreatic cancer cells [6]. shRNA silencing of zinc transporter ZIP4 has been shown to inhibit tumor growth and extend the survival of nude mice with pancreatic cancer xenografts [9]. In addition to re-expressing tumor suppressor genes, gene therapy strategies can also deliver suicide genes to tumor cells, which encode for drug-activating enzymes. Pancreatic cancer cells transfected with cytosine deaminase, which converts 5-fluorocytosine (5-FC) to its active form fluorouracil (5-FU), showed decreased tumor growth when subsequently treated with 5-FU [12]. However, despite strong potential, the success of gene therapy agents, especially in clinical studies, has been limited, perhaps because of the complex nature of pancreatic tumors. Simultaneous targeting of multiple gene mutations may be necessary to overcome the effects of extensive crosstalk.

The epidermal growth factor receptor (EGFR) inhibitor erlotinib is FDA approved in combination with gemcitabine to treat metastatic pancreatic cancer based on clinical trial results that showed significantly increased overall survival and progression-free survival compared to gemcitabine monotherapy in this patient population $[10,13]$. EGFR, involved in cancer growth and metastasis, promotes epithelial-to-mesenchymal transition for decreased cell adhesion and increased cell migration. Overexpression of EGFR has been shown to be common in pancreatic cancer and may indicate the potential aggressiveness of the cancer [14]. Also, several studies have utilized carbon nanotubes for imaging as a significantly better contrast agent [15].

Other investigational therapeutic agents include monoclonal antibodies against targets such as EGFR, vascular endothelial growth factor receptor (VEGFR), mucin 1 (MUC1), and mesothelin. Antibody therapies have the potential to inhibit tumor growth and angiogenesis and provide a method of treating otherwise resistant cancers. Oncolytic viral therapies, often using adenovirus and herpes simplex virus, are engineered to replicate selectively in tumor cells, targeting the cells for lysis, cell-to-cell fusion, or immune response.

\section{Delivery vehicles for pancreatic cancer therapies}

Commonly used delivery vehicles for cancer therapies include liposomes and nanoparticles. However, there are few clinical trials using these newer delivery methods in pancreatic cancer. Most current pancreatic cancer chemotherapies work systemically and thus are subject to a number of physiologic and chemical limitations that decrease efficacy. Unfavorable pharmacokinetics and poor targeting of cancer cells among other issues significantly affect the ability of these drugs to provide greater clinical benefit, and toxicity and side-effects limit acceptable dosages. The newer delivery vehicles not only target specific organs but also offer increased drug solubility, protection against degradation and elimination, decreased toxicity, and reduced resistance [5].

Liposomes are vesicles composed of single or multiple phospholipid bilayers that can be loaded with a variety of content including genetic materials and chemotherapeutic drugs. Liposomes can improve drug solubility and stability, are biodegradable, and exhibit low toxicity. They have already proven to be viable clinically, with several FDA-approved liposome formulations in existence for cancer treatment. Liposomes have been used for in vitro gene transfection to protect antisense oligonucleotides, siRNA, and shRNA from degradation and improve transfection efficiency and targeting. For example, liposomal delivery of both pancreatic and duodenal homeobox 1 (PDX-1) and ZIP4-targeted shRNA was shown to inhibit tumor growth in immunodeficient mice [16, unpublished data]. The addition of surface ligands can help target liposomes to cells of interest, thereby helping to reduce the toxicity of therapeutic agents and enrich concentrations in target tissues. Liposomes can also be pegylated to increase stability and prolong the circulation time of a drug. In a study by Cosco and colleagues, pegylated liposomes loaded with gemcitabine increased survival and reduced tumor growth and toxicity in severely compromised immunodeficient (SCID) xenograft mouse models of pancreatic cancer compared to controls treated with standard gemcitabine [17]. Improved liposomal delivery efficiency may further allow 
lower concentrations of drugs to be used for the same effect, prolong circulation times, improve drug internalization, and decrease unwanted toxic side-effects.

The benefits of nanoparticles are a large surface-to-mass ratio and the ability to bind to a number of substances, provide sustained release of drugs, and improve drug circulation and concentration. Stearoyl gemcitabine nanoparticles have been found to enhance the effect of gemcitabine on pancreatic cancer cells in vitro, and suppress tumor growth more effectively than normal gemcitabine in a mouse model [18]. Nanoparticles can also be targeted to specific locations through the addition of monoclonal antibodies or use of magnetic guidance. Recently, carbon nanotubes have emerged as an exciting potential modality for drug delivery and imaging. They are able to absorb a large amount of drug, which can be slowly released, and their stable structure can be modified for improved targeting of therapies. Carbon nanotube-delivered cisplatin modified to target EGFR-expressing cells was found to selectively kill head and neck squamous carcinoma cells (HNSCCs) [19].

\section{Improved targeting in pancreatic cancer therapy}

To improve the targeting of new therapeutic agents to pancreatic tumor cells, the unique expression profile of pancreatic cancer cells can be exploited to differentiate normal, healthy cells from cancer cells. Liposomes, nanoparticles, and carbon nanotubes can be modified with monoclonal antibodies or ligands to allow the therapeutic agents to be directed towards specific cells, thus increasing the accumulation of drugs in the target organ and decreasing toxicity to normal cells. Potential targets that can be used include EGFR, urokinase plasminogen activator receptor (UPAR), transferrin receptor, HER-2, CA125, mesothelin, and MUC1. These potential delivery targets have been shown to be overexpressed in pancreatic cancer cells [5]. However, because of the heterogeneity of pancreatic cancer and the variable expression profiles among pancreatic cancer cells, further research is warranted to identify more effective targets for drug delivery.

\section{Challenges and future work}

Increasing evidence suggests that the tumor microenvironment may play a critical role in pancreatic cancer cell resistance and explain the disparity between preclinical and clinical study results. Dense desmoplastic stroma and poor perfusion of the tumor prevent drugs from properly penetrating pancreatic tumors. Inhibition of hedgehog signaling has been found to decrease stromal tissue and increase perfusion of the tumor, thus improving survival in a mouse model [7]. For novel therapeutics to be successful, it is likely that there will be a need for a greater understanding of the microenvironment and how it can be overcome for better drug delivery.

Recent advancements in the development of therapeutic agents and delivery modalities for improved targeting, efficacy, and clinical outcomes in pancreatic cancer are extremely promising. However, because the data are mostly preclinical and the clinical trials are few, more research is necessary to further refine these methods, confirm their safety, and improve delivery specificity. Even if targeted delivery is accomplished and the drug enters the cell, it is possible that there will be a response failure. Therefore, a greater understanding of pancreatic cell biology is also crucial since the presence of multiple mutations may make single-target treatments insufficient. Emerging targeted therapeutic approaches may be exciting, viable opportunities that will tangibly improve the management of this devastating disease.

\section{Funding}

The Authors received an honorarium from the Publisher.

\section{References}

1. Jemal A, Bray F, Center MM et al. Global cancer statistics. Ca Cancer J Clin 2011;61(2):69-90.

2. Siegel R, Naishadham D, Jemal A. Cancer statistics, 2012. CA Cancer J Clin 2012;62(1):10-29.

3. Li D, Xie K, Wolff R, Abbruzzese JL. Pancreatic cancer. Lancet 2004;363(9414):1049-57.

4. Conroy T, Desseigne F, Ychou M et al; Groupe Tumeurs Digestives of Unicancer; PRODIGE Intergroup. FOLFIRINOX versus gemcitabine for metastatic pancreatic cancer. N Engl J Med 2011;12;364(19):1817-25.

5. Yu X, Zhang Y, Chen C, Yao Q, Li M. Targeted drug delivery in pancreatic cancer. Biochim Biophys Acta 2010;1805(1):97-104.

6. Guillermet-Guibert J, Davenne L, Pchejetski D et al. Targeting the sphingolipid metabolism to defeat pancreatic cancer cell resistance to the chemotherapeutic gemcitabine drug. Mol Cancer Ther 2009;8(4): 809-20.

7. Olive KP, Jacobetz MA, Davidson CJ et al. Inhibition of hedgehog signaling enhances delivery of chemotherapy in a mouse model of pancreatic cancer. Science 2009;324(5933):1457-61.

8. Aoki K, Yoshida T, Sugimura T, Terada M. Liposomemediated in vivo gene transfer of antisense K-ras construct inhibits pancreatic tumor dissemination in the murine peritoneal cavity. Cancer Res 1995;55(17): 3810-16.

9. Li M, Zhang Y, Bharadwaj U et al. Down-regulation of ZIP4 by RNA interference inhibits pancreatic cancer growth and increases the survival of nude mice with pancreatic cancer xenografts. Clin Cancer Res 2009;15(19): 5993-6001.

10. Moore MJ, Goldstein D, Hamm J et al; National Cancer Institute of Canada Clinical Trials Group. Erlotinib plus gemcitabine compared with gemcitabine alone in patients with advanced pancreatic cancer: a phase III trial of the National Cancer Institute of Canada Clinical Trials Group. J Clin Oncol 2007;25(15):1960-6.

11. Bouvet M, Bold RJ, Lee J et al. Adenovirus-mediated wild-type p53 tumor suppressor gene therapy induces apoptosis and suppresses growth of human pancreatic cancer. Ann Surg Oncol 1998;5(8):681-8. 
12. Evoy D, Hirschowitz EA, Naama HA et al. In vivo adenoviral-mediated gene transfer in the treatment of pancreatic cancer. J Surg Res 1997;69(1): 226-31.

13. Strimpakos A, Saif MW, Syrigos KN. Pancreatic cancer: from molecular pathogenesis to targeted therapy. Cancer Metastasis Rev 2008;27(3):495-522.

14. Einama T, Ueda S, Tsuda $\mathrm{H}$ et al. Membranous and cytoplasmic expression of epidermal growth factor receptor in metastatic pancreatic ductal adenocarcinoma. Exp Ther Med 2012;3(6):931-6.

15. De la Zerda A, Zavaleta C, Keren $S$ et al. Carbon nanotubes as photoacoustic molecular imaging agents in living mice. Nat Nanotechnol 2008;3(9):557-62.

16. Liu S, Ballian N, Belaguli NS et al. PDX-1 acts as a potential molecular target for treatment of human pancreatic cancer. Pancreas 2008;37(2): 210-20.

17. Cosco D, Bulotta A, Ventura $M$ et al. In vivo activity of gemcitabine-loaded PEGylated small unilamellar liposomes against pancreatic cancer. Cancer Chemother Pharmacol 2009;64(5):1009-20.

18. Sloat BR, Sandoval MA, Li D et al. In vitro and in vivo anti-tumor activities of a gemcitabine derivative carried by nanoparticles. Int J Pharm 2011;409(1-2):278-88.

19. Bhirde AA, Patel V, Gavard J et al. Targeted killing of cancer cells in vivo and in vitro with EGF-directed carbon nanotube-based drug delivery. ACS Nano 2009;3(2): 307-16. 